\title{
Sur l'usinabilité des composites à matrices polymères renforcée par des fibres
}

\author{
Francisco Mata Cabrera ${ }^{1, a}$, Issam Hanafi ${ }^{2}$, Abdellatif Khamlichi ${ }^{2}$, \\ AbDallah JabBouri ${ }^{3}$ et Mohamed BezZazI ${ }^{3}$ \\ 1 École universitaire polytechnique d'Almaden, Place Manuel Meca, 1, 13400, Almaden, Ciudad Real, Espagne \\ 2 Faculté des Sciences, M'hannech II, BP 2121, Tétouan, Maroc \\ 3 Faculté des Sciences et Techniques, Ancienne Route de l'Aéroport, Km 10, BP 416, Tanger, Maroc
}

Reçu le 18 mars 2009, accepté le 26 février 2010

\begin{abstract}
Résumé - Une revue bibliographique étendue est réalisée sur le thème de l'usinabilité des composites à matrice polymère renforcée par des fibres, dans le but de fournir des résultats et conclusions sur l'usinage des matériaux composites à matrice thermoplastique et à fibres courtes. Un rappel sur l'état de l'art dans ce domaine, sur les spécificités liées à l'usinage des composites organiques et sur les différents modèles ayant été introduits pour prédire les paramètres de coupe a été effectué. Les paramètres affectant l'usinabilité proprement dite des composites ont été revus, ensuite une riche bibliographie sur le sujet est présentée. Enfin les critères d'usinage ont été présentés sous forme synthétique en considérant les différentes contributions développées dans ce domaine et, de manière presque exhaustive, toutes les références bibliographiques significatives ayant traité de ce sujet.
\end{abstract}

Mots clés : Revue bibliographique / usinabilité / chariotage / composite polymérique renforcés par des fibres / état de l'art / paramètres de coupe / critères d'usinage

\begin{abstract}
On machinability of fiber reinforced polymeric composites. A literature review about machinability of fiber reinforced polymeric composites is given, in order to provide results and conclusions on the machining of composite materials with thermoplastic matrix and short fibers. The significant contributions to the current state-of-the-art are surveyed by considering the machining aspects involved in organic composites. The various models which have been introduced to predict the cutting parameters are also reviewed. Then, the parameters affecting machinability of composites are thoroughly examined. The various criteria for machining purposes are after that presented in a synthetic form where analysis of the various contributions developed in this field is given. Finally, a list of issues requiring further research is highlighted.
\end{abstract}

Key words: Review / machinability / turning / fiber reinforced polymeric composites / state-of-the-art / cutting parameters / criteria for machining

\section{Introduction}

L'usinage est un procédé de fabrication au cours duquel un outil de coupe est utilisé pour éliminer l'excès de matière afin d'obtenir la forme et les dimensions spécifiées d'une pièce mécanique. Le chariotage est l'une des opérations d'usinage les plus utilisées dans l'industrie pour produire une grande variété de composantes

\footnotetext{
a Auteur pour correspondance:

Francisco.MCabrera@uclm.es
}

mécaniques qui répondent aux divers besoins dans le domaine de la fabrication. Très souvent, les surfaces travaillant dans les conditions de contact mécanique pour diverses applications tribologiques sont obtenues en utilisant ce procédé [1].

Actuellement le procédé de chariotage n'est plus réservé aux matériaux métalliques et sert aussi à élaborer des matériaux composites à matrices organiques. L'intérêt porté aux techniques d'usinage des matériaux organiques a ainsi augmenté de manière significative. La majorité des travaux de recherche qui sont développés dans ce domaine 
portent essentiellement sur la caractérisation du procédé de chariotage afin de maîtriser son emploi à l'échelle de la fabrication industrielle, l'objectif étant d'obtenir des composants mécaniques avec des caractéristiques dimensionnelles rigoureusement contrôlées au moindre coût possible. Parmi les matériaux organiques qui sont élaborés par chariotage, on trouve les matériaux plastiques renforcés avec des fibres de verre (GFRP's) et les matériaux plastiques renforcés avec des fibres de carbone (CFRP's). Les conditions spéciales d'utilisation de ces composites requièrent des précautions particulières durant leur processus de fabrication. Remarquons à cet égard que le chariotage des matériaux composites, constitués d'une matrice polymère contenant des renforts est très différent de celui des métaux et alliages [2]. Les connaissances théoriques et expérimentales acquises au niveau des métaux ne s'appliquent pas telles quelles pour ce type de matériaux composites; car ces derniers sont en général anisotropes et hétérogènes, du fait par exemple, qu'ils sont préparés sous forme stratifiée ou bien extrudés avant d'envisager de les usiner.

Les matériaux composites contiennent deux phases, avec des propriétés mécaniques et thermiques très différentes. Des interactions complexes apparaissent entre la matrice et le renfort pendant le processus de fabrication par chariotage de sorte qu'elles affectent de manière particulière les caractéristiques d'usinage de ces matériaux en comparaison avec ceux constitués par une seule phase tels que les métaux [3-6]. Le comportement des matériaux composites durant l'usinage dépend des propriétés des fibres et de leur orientation, des propriétés de la matrice et du volume relatif de renfort. Pendant l'usinage, l'outil de coupe trouve alternativement matrice et fibres avec une réponse à l'action d'usinage qui est différente pour chacun de ces deux types de matériaux. Le processus de formation du copeau peut être dû à la rupture, au cisaillement, ou à une combinaison de ces deux mécanismes selon aussi l'orientation de la fibre et de la géométrie de l'outil. Le caractère abrasif des fibres celui de la matrice, surtout si celle-ci est thermodurcissable, peut engendrer des usures prématurées de l'outil, pour cela il est recommandé d'utiliser des outils de diamant polycrystallin (PCD) [7].

Comme la matrice a une faible conductivité thermique comparée à celles des métaux et d'autres matériaux inorganiques, un échauffement de la pièce peut se produire pendant l'usinage. Ce phénomène peut engendrer une instabilité dimensionnelle et favoriser l'apparition de vibrations nuisibles. Par ailleurs, il est nécessaire de tenir compte de la présence d'un lubrifiant car celui-ci peut être absorbé par la matrice provoquant ainsi une variation locale des propriétés, ce qui pourrait conduire à des instabilités. Pour cette raison, il est souvent recommandé de n'utiliser aucun lubrifiant lors du chariotage des matériaux organiques.

Un dégagement excessif de chaleur au cours d'usinage peut entraîner un risque de dégradation thermique, surtout si la température produite par la coupe dépasse la température de transition vitreuse $T_{\mathrm{g}}$. Globalement, ce risque provoque la carbonisation des matrices thermodurcissables et la fusion des matrices thermoplastiques, ce qui peut présenter un danger pour les opérateurs : inflammation, échappement de gaz toxiques. Pour réduire le risque, les tours CNC doivent être équipés de vitres protectrices. Afin d'éviter aussi tout problème d'intoxication par inhalation de gaz, lorsque la nature des gaz susceptibles de se dégager n'est pas bien connue, les opérateurs doivent porter des masques de protection. Dans tous les cas il faut surveiller l'opération de chariotage et ne pas l'automatiser en l'absence d'opérateur.

L'usinage des petites pièces de géométrie cylindrique est important dans la fabrication des composants des machines et des robots à une époque où la tendance est vers la miniaturisation. Lu et Yoneyama décrivent un système de micro-chariotage qui a nécessité une optimisation des outils de coupe afin de mener correctement le processus de chariotage [8]. Picard et al. proposent un nouveau système de fabrication d'outils de coupe de petite taille par la technologie «sputtering». Ils ont réussi à fabriquer des outils à base de carbure tungstène, acier rapide et cristaux de diamant avec différentes formes et géométries, qu'ils ont essayées avec de bons résultats lors du processus de microchariotage cylindrique [9].

Les matériaux composites de matrice polymère présentent une vaste variété de modes de défaillance qui comprennent la rupture des fibres, l'écoulement plastique de la matrice, le délaminage, etc. [10]. Des recherches ont mis en évidence que la texture superficielle et les défauts de fabrication agissent sur les charges statiques et dynamiques de service et affectent la résistance à la fatigue [11]. Par conséquent, on doit considérer attentivement l'état de qualité des surfaces usinées et veiller à les contrôler en vue d'améliorer la performance des composants fabriqués par chariotage. Parmi les travaux consacrés à cet aspect des choses, il convient de citer des études s'intéressant à la prédiction des forces de coupe en observant les modes de rupture rencontrés lors de la séparation du copeau [12-15].

\section{Le chariotage des composites}

Les propriétés des matériaux ont une influence considérable sur les conditions rencontrées lors de l'opération d'usinage (Fig. 1). La figure 2 montre les différents facteurs qui affectent l'usinabilité des matériaux; en particulier, ceux qui sont relatifs aux propriétés du matériau, aux paramètres et aux outils de coupe. L'usinage fait référence en fait à la facilité avec laquelle le matériau peut se prêter à cette opération lorsque des outils de coupe et des paramètres fonctionnels appropriés ont été sélectionnés [16]. L'aptitude relative permettant à un matériau d'être usiné est habituellement exprimée par l'intermédiaire d'un coefficient appelé indice d'usinage. Il s'agit en fait d'une échelle qui permet de contrôler ce procédé. Cette échelle est établie en procédant à des comparaisons entre différents types de matériaux afin d'identifier le degré de facilité qui décrit les conditions d'usinage d'un matériau donné. Si dans le cas 


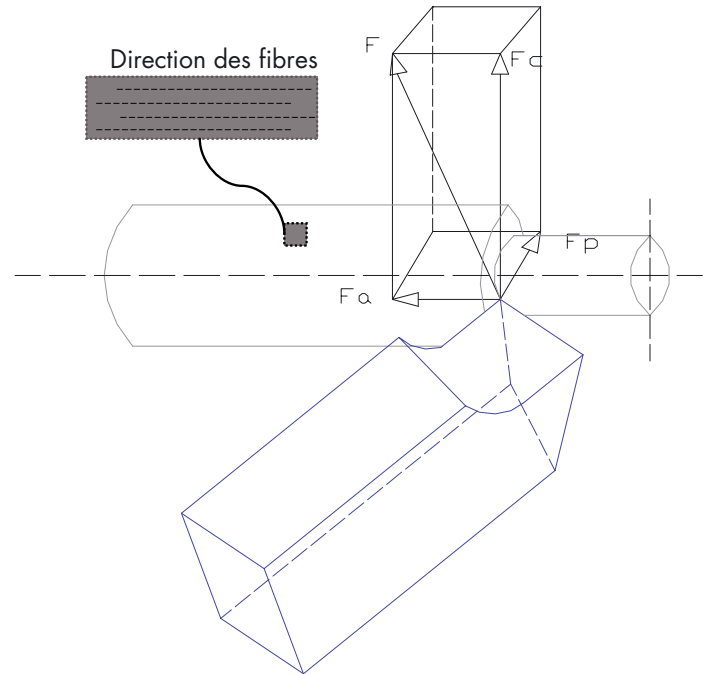

Fig. 1. Interaction outil de coupe-pièce usinée; composantes de la force d'usinage $F: F_{\mathrm{a}}$ : Force d'avance, $F_{\mathrm{p}}$ : Force de pénétration, $F_{\mathrm{c}}$ : Force de coupe.

des matériaux conventionnels, un indice d'usinage est admis de manière unanime depuis longtemps, dans le cas des matériaux plastiques renforcés il n'existe aucun critère qui soit universellement reconnu. Comme le laisse entrevoir ce travail de recherche, il est nécessaire d'approfondir l'analyse des mécanismes de chariotage propres aux matériaux composites afin d'établir des règles et des critères qui serviront à éditer de telles références largement attendues dans le domaine.

Diverses recherches ont abordé l'étude de l'usinage des matériaux plastiques de nature thermostable lorsqu'ils sont renforcés avec des fibres. An et al. ont étudié les caractéristiques du processus de coupe [17], en prêtant une attention particulière à l'influence due au matériau et à la géométrie de l'outil. Ferreira et al. ont analysé différents aspects liés à l'usinage des matériaux composites renforcés avec des fibres de carbone [18]. Hocheng et Tsao ont effectué des séries d'essais sur des matériaux composites de matrice polymère renforcée avec des fibres de carbone. En observant les caractéristiques du copeau, ils ont évalué le degré d'usinabilité de ces matériaux en fonction de la direction des fibres et des paramètres de coupe. Ils ont constaté en particulier que la composante de la force de coupe qui est parallèle aux fibres était plus petite que celle qui leur est perpendiculaire [19,20], ces divers résultats ont été établis en utilisant le modèle de Koplev [21]. Chang a effectué une étude sur l'usinage des matériaux plastiques renforcés avec des fibres de verre en utilisant des outils de carbure de tungstène et a développé des modèles de prédiction des forces de coupe [22].

\section{Modèles de coupe des composites}

La première recherche théorique effectuée sur l'usinage des matières plastiques renforcées avec des fibres est due à Everstine et Rogers [23]. Ces chercheurs ont formulé un modèle permettant la prédiction de la force minimale de coupe pour des fibres parallèles, autrement dit admettant l'orientation $0^{\circ}$.

Presque 10 années après Koplev a fait des essais en adoptant une configuration de coupe qui va du cas parallèle au cas orthogonal à la direction des fibres de renfort [24]. Il a travaillé sur du CFRP charioté par des outils à une arête de coupe unique. En analysant le processus de formation du copeau et l'état de la surface usinée, il a trouvé que la qualité de la surface réalisée par l'usinage dépendait considérablement de l'orientation des fibres. Les surfaces étaient ainsi plus lisses lorsque la direction de coupe était parallèle à la direction des fibres. La rugosité augmente lorsque la direction de l'usinage s'approche de la configuration perpendiculaire; c'est à $-45^{\circ}$ et à $+45^{\circ}$ que les défauts de rugosité sont les plus critiques.

Koplev a mis en évidence aussi le fait que la force de friction engendrée par le contact outil/fibre était à l'origine de la rupture de celle-ci par traction.

Dans un travail postérieur, Koplev et al. ont étudié la relation entre les forces de coupe, le mécanisme de formation du copeau et la géométrie de l'outil [21]. Ils ont trouvé que la force de coupe principale était proportionnelle à la profondeur de passe et diminuait légèrement lors de l'augmentation de l'angle de coupe. Ils ont mis ainsi en évidence qu'une grande réduction se produisait dans la force d'avance lorsque cet angle augmente de 3 à $15^{\circ}$, le fait d'augmenter cet angle, on augmente le taillant de l'outil ce qui fait chuter la force d'avance. Ils ont suggéré, comme explication, le fait que la force était proportionnelle à la surface de contact entre l'outil et la pièce. Ils ont aussi démontré que la force de coupe était indépendante de l'usure de l'outil et que l'accroissement de la force de coupe était dû à l'augmentation de la force de friction entre l'outil et la pièce, résultant à son tour de la force d'avance.

Sakuma et Seto ont effectué des essais de chariotage unidirectionnel sur des tubes de GFRP dans le but d'étudier les effets de l'orientation des fibres de verre sur l'usure de l'outil et sur les forces de coupe. Ils ont observé des processus de formation de copeau qui étaient semblables à ceux de Koplev. Ils ont constaté également que pour les petits angles définissant l'orientation des fibres, celles-ci d'abord pliées sous la progression de l'outil avant de casser par traction. Pour les grands angles qui définissent l'orientation des fibres, celles-ci subissaient une déformation élastique assimilable à de la flexion puis étaient cisaillées par rupture fragile. L'explication avancée par Sakuma et Seto est la variation affectant les forces de coupe en fonction de l'orientation des fibres par le fait que la résistance tangentielle des fibres de verre est plus petite que la résistance normale [25].

Takeyama et Iijima ont étudié le processus de formation du copeau en chariotant des matériaux plastiques renforcés avec des fibres de verre pour différents angles d'orientation des fibres. Ils ont proposé un modèle, pour prédire les forces de coupe, basé sur la théorie d'énergie minimale de Merchant. Ils ont vérifié que, pour un angle d'orientation de $0^{\circ}$, la rupture avait lieu dans la direction 


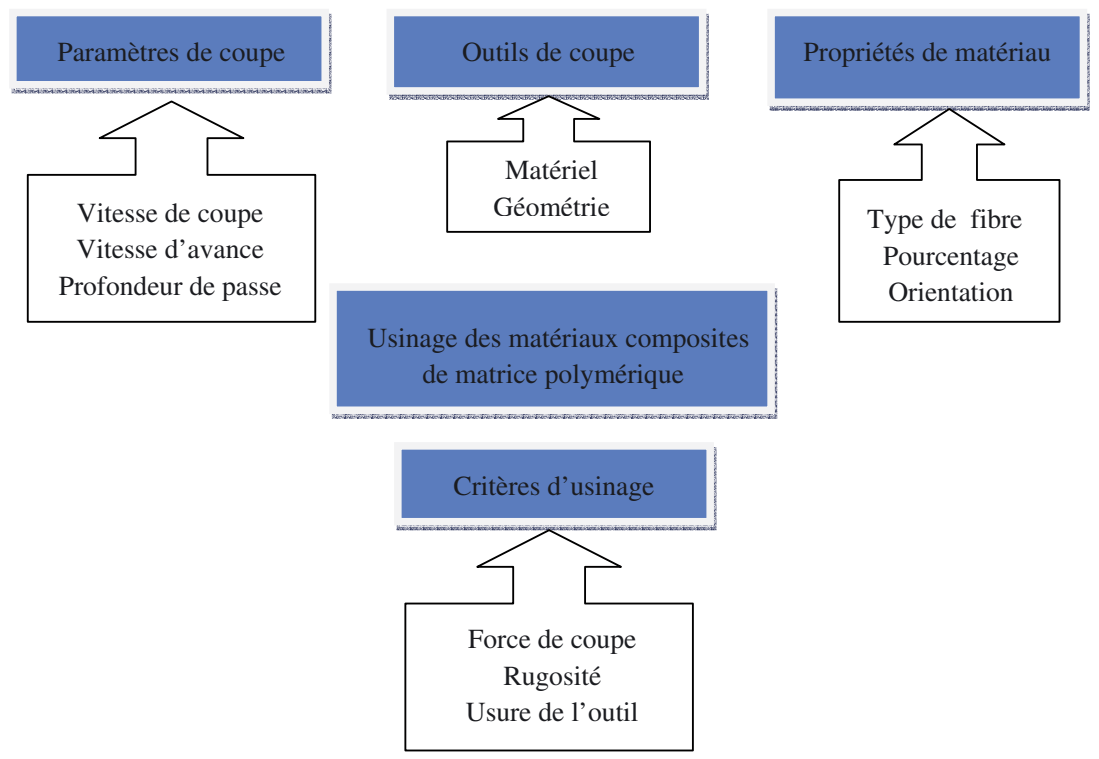

Fig. 2. Facteurs qui affectent l'usinage.

de la fibre, tandis que pour un angle de $90^{\circ}$ on produisait la rupture du copeau [26].

Arola et Ramulu ont développé une analyse par modèle éléments-finis $2 \mathrm{D}$ pour simuler le processus de formation du copeau dans le cas des composites unidirectionnels [11]. La séparation du copeau a été modélisée en utilisant un critère de traction critique basé sur la résistance des constituants du matériau composite. Le modèle a été validé en utilisant des mesures expérimentales des forces de coupe. Les valeurs mesurées pour la force de coupe s'accordaient bien avec le modèle, mais les prédictions concernant les valeurs de la force d'avance étaient incorrectes, à cause des difficultés liées à la définition du plan de rupture.

Ramesh et al. ont proposé un modèle éléments-finis pour l'usinage des FRP's unidirectionnels qui est basé sur la théorie de la plasticité anisotrope. Le matériau a été modélisé comme étant homogène et l'outil de coupe comme étant parfaitement élastique. En utilisant les propriétés physiques et mécaniques, obtenues expérimentalement, ils ont modélisé le processus de formation du copeau de différents matériaux composites. Parmi ceux-ci, il y a les matériaux plastiques renforcés avec des fibres de verre (GFRP's), avec des orientations de $0^{\circ},+45^{\circ}, 90^{\circ}$ et $135^{\circ}$, [27]. Ils ont constaté des variations dans les forces de coupe en fonction de l'angle d'orientation des fibres qui étaient semblables à celles obtenues par Bhatnagar [2]. Ils ont observé également que le cisaillement de la matrice était la cause de rupture prédominante.

Ribeiro et Coppini ont proposé un système d'acquisition afin d'optimiser le processus de coupe et les coûts de production. Ce système intègre le matériau, l'outil de coupe, la machine-outil et les conditions de coupe utilisées [28].

Mahdi et Zhang ont proposé un algorithme tridimensionnel adaptatif à base d'éléments-finis qui permettait de modéliser la fibre et la matrice comme une cellule composite [29]. Ils ont aussi présenté un modèle de coupe de une à deux dimensions afin de prédire le comportement de la force de coupe des matériaux composites par rapport à l'angle d'orientation des fibres [30].

La sélection correcte de l'outil et des paramètres de coupe (vitesse de coupe, vitesse d'avance et profondeur de passe) permet de réduire significativement les coûts. L'effet de la géométrie de l'outil de coupe et des paramètres a été évalué par Wang et Zhang [31,32], en analysant la formation du copeau, les forces de coupe et la rugosité. Tous ces aspects dépendent directement de l'orientation des fibres dans le matériau.

Des études expérimentales de l'usinage des matériaux plastiques renforcés avec des fibres de carbone, effectuées par différents chercheurs démontrent que la formation du copeau dépend fortement de l'orientation des fibres [31-33]. En plus de l'orientation des fibres, le matériau et la géométrie de l'outil de coupe ont aussi une influence significative sur les surfaces usinées, sur la formation du copeau, sur les forces de coupe et sur l'usure de l'outil lui-même $[4,34]$.

Ramulu et al. ont mené à bien des études de caractérisation de la coupe orthogonale des matériaux composites renforcés avec des fibres de verre et analysé les effets de l'orientation des fibres, des paramètres de coupe, de la géométrie de l'outil et de sa qualité superficielle $[35,36]$.

\section{Paramètres affectant l'usinabilité des composites}

\subsection{Propriétés des matériaux}

Les propriétés mécaniques du matériau qui influencent le plus l'usinage sont la dureté et la résistance à 
l'usure [37]. En augmentant la dureté, l'usure abrasive augmente et la vie de l'outil est réduite. En augmentant la résistance du matériau, les forces de coupe augmentent au même titre que l'énergie spécifique et la température de coupe, rendant ainsi le matériau plus difficile à usiner.

Les propriétés physiques et thermiques des fibres et de la matrice polymère sont différentes. Celles du composite dépendent du type de fibres, du contenu ou du pourcentage de fibres et de leur orientation, et de la variabilité dans la matrice elle-même [38], d'où la grande influence que ces paramètres peuvent avoir sur le comportement tribologique de ces matériaux. Les matériaux plastiques renforcés ont un comportement extrêmement abrasif quand ils sont soumis à des processus de formation de copeau. La résistance mécanique importante des fibres peut être à l'origine d'une usure excessive de l'outil de coupe et d'un dommage dans la matrice polymérique.

Rahman et al. ont développé une étude sur l'usinage des matériaux composites PEEK renforcés avec des fibres de carbone et ont conclu que l'état lisse de la surface de la pièce produite est dû à la matrice polymère et la géométrie de l'outil de coupe [6].

\subsection{Paramètres fonctionnels de coupe}

En parlant des paramètres fonctionnels de coupe, on se réfère en général à la sélection de la vitesse de coupe, de la vitesse d'avance et de la profondeur de passe. Il est important aussi de considérer la nécessité d'utiliser ou non et de sélectionner de manière adéquate, le cas échéant, un lubrifiant. Plusieurs études ont été dédiées aux effets des paramètres de coupe sur l'opération de tournage $[37,40]$. Ces études ont corroboré le fait que pour les matériaux composites il n'existait pas de référence universelle. Chaque matériau, selon le type et le pourcentage des fibres de renforcement, présente des valeurs de vitesse coupe et de vitesse d'avance qui lui sont appropriées lorsqu'on désire une finition superficielle optimale et une diminution de l'usure de l'outil.

Il est nécessaire donc de concevoir de nouvelles expériences pour garantir des résultats satisfaisants susceptibles de qualifier le processus d'usinage [41] ; en particulier, les relations expérimentales qui permettent de corréler les paramètres de coupe avec les caractéristiques dimensionnelles finales, puisque celles-ci constituent le centre d'intérêt pour l'industrie [42].

L'utilisation d'un lubrifiant pendant le processus de chariotage peut entraîner l'absorption de ce produit par la matrice polymère et produire ainsi des modifications dimensionnelles des pièces et celles de leurs propriétés mécaniques. Par conséquent, il est préférable d'exécuter le processus d'usinage sans faire appel à la réfrigération. Cette situation a permis de relancer une activité de recherche présentant un grand intérêt pratique afin de savoir s'il faut usiner à sec ou non [43].

Sardinas et al. qui ont eu recours à des algorithmes génétiques, ont présenté une étude d'optimisation des paramètres de coupe afin d'usiner de manière optimale des composites laminés [44].

\subsection{Outil de coupe}

Les outils de coupe les plus utilisés dans l'usinage des matériaux composites de matrice polymère sont le diamant polycrystallin (PCD) et les carbures de tungstène (K10). Différents travaux effectués sur l'usinage des matériaux plastiques renforcés avec des fibres (FRP's) ont conclu que les outils PCD offraient les meilleurs résultats. Toutefois, le principal inconvénient des outils PCD est leur coût qui peut atteindre jusqu'à dix fois celui des carbures. Récemment, des outils avec revêtement de diamant ont été mis en œuvre et constituent une alternative sérieuse aux PCD.

La coupe des matériaux plastiques renforcés avec des fibres est difficile étant donné le problème de délaminage du matériau composite qui risque de se poser et la courte durée de vie de l'outil [44]. Ceci oblige de recourir à des outils élaborés avec des matériaux de haute résistance à l'usure et dont la ténacité et la dureté à chaud sont élevées (carbures de tungstène, diamant polycrystallin et revêtement de diamant) [16].

La sélection de l'outil et des paramètres de coupe est très importante dans le processus d'usinage $[5,46]$. C'est le matériau de l'outil qui sert à développer les forces de coupe (force d'usinage, force spécifique de coupe, puissance de coupe) comme il gouverne largement l'état de finition de la surface usinée. Les carbures de tungstène sont formulés à partir de carbure de tungstène $22 \#(\mathrm{WC})$ et ils sont fabriqués au moyen de techniques métallurgiques à base de poudres dans lesquelles on utilise le cobalt $22 \#(\mathrm{Co})$ comme liant [16].

La dureté extrême du diamant, sa conductivité thermique importante et son petit coefficient de friction, font de lui un matériau idéal pour les outils de coupe. Le diamant a été utilisé largement sous forme d'inséré polycrystallin sur l'outil de carbure de tungstène (PCD) et aussi sous forme de diamant naturel $[47,48]$.

Le diamant polycrystallin est fabriqué au moyen de la sintérisation de poussières fines de cristaux de diamant granulé à hautes températures et pressions. L'utilisation des outils de diamant polycrystallin (PCD) a connu une croissance importante étant donné sa durée de vie et sa finition superficielle importante en comparaison avec les outils de carbures de tungstène [48], en plus les PCD ont un plus petit rayon d'acuité d'arête.

Chambers et Bishop ont effectué des essais sur l'usinage des composites Carbone/Epoxy et Carbone/PEEK en utilisant des outils en carbures de tungstène et en diamant polycrystallin (PCD). Ils ont conclu que l'outil PCD fournit les meilleurs résultats en termes de qualité d'usinage [49]. Une autre étude due à Jahanmir permet de corroborer ce résultat à savoir qu'une meilleure qualité de surface est toujours obtenue avec les outils de coupe PCD [50].

La synthèse du diamant à basses pressions par déposition chimique en phase vapeur (CVD) permet d'utiliser le diamant sous forme de couches déposées sur l'outil en vue des applications d'usinage [51-53]. Le diamant CVD peut être produit en diffusant un gaz hydrocarburé dans l'hydrogène par chauffage autour de $2000{ }^{\circ} \mathrm{C}$ et en 
déposant le diamant sur un substrat adéquat [47,54]. Les propriétés du diamant CVD sont très influencées par la structure du grain et par conséquent dépendent des conditions de synthèse [55]. Il existe des difficultés d'adhésion de la couche de diamant au substrat et des écarts de dilatation thermique entre le diamant et les matériaux substrat [56]. Les fabricants d'outils CVD ont développé différents processus pour déposer le diamant sous forme de cristaux de différentes tailles et épaisseurs, depuis peu on sait atteindre des tailles qui vont du micron jusqu'à plusieurs millimètres selon l'application désirée [54].

Les outils de diamant CVD possèdent de bonnes propriétés tel qu'un faible coefficient de frottement (inférieur à 0,05 ), une conductivité thermique adéquate (au-dessus de $2200 \mathrm{~W} \cdot \mathrm{mK}^{-1}$ ), une grande dureté à haute température (6000-9000 HV) et une excellente stabilité chimique [54]. Puisque le diamant CVD a de meilleures propriétés thermiques et chimiques en comparaison avec le PCD, son risque de rupture est plus faible et par conséquent on peut l'utiliser pour des opérations de finition dans des alliages non ferreux et les matériaux modernes non métalliques [57]. Dans le cas des matériaux composites de matrice polymérique, le diamant PCD a un effet positif car, du fait de sa grande dureté, il résiste facilement à l'attaque abrasive, tandis que la surface lisse du diamant affiche une tendance réduite à l'adhésion contre les polymères [58].

L'usinage des matériaux composites de matrice polymère demande une adhésion excellente du recouvrement de diamant. Si le matériau polymérique est collé au bord de l'outil, les forces de coupe augmentent et par conséquent des tensions additionnelles sont induites dans le recouvrement. Ceci provoque une augmentation de la rugosité et peut conduire carrément au délaminage de la couche.

Divers travaux ont été publiés dans la littérature où l'étude du comportement des outils de coupe revêtus de diamant lors des opérations d'usinage a été abordée, dans le cas des alliages d'aluminium, des matériaux composites à matrice métallique, du graphite ou bien des matières plastiques renforcées avec des fibres. Reineck et al. ont présenté un travail dont le but était d'évaluer l'effet induit par des outils de coupe revêtus avec du diamant et produits par la technique HCDCA (High Current DCArc technique). Ils ont effectué des essais sur plusieurs configurations d'usinage en utilisant des éprouvettes de divers matériaux, depuis les alliages $\mathrm{Al}-\mathrm{Si}$, aux matériaux composites à matrice métallique et aux matières plastiques renforcées avec des fibres de verre. Ces auteurs ont démontré qu'un meilleur comportement, caractérisé par une grande résistance à l'usure et une très bonne qualité de surface, est obtenu en comparaison avec les outils classiques en carbures sans recouvrement [47].

Par ailleurs, un travail expérimental intéressant a été effectué par Davim sur l'usinage des matériaux composés de matrice métallique (A356/SiC/20p). L'auteur a comparé le comportement des outils en diamant polycrystallin (PCD) à celui des outils revêtus de diamant produit par déposition chimique en phase vapeur (CVD). Les comparaisons ont porté sur la durée de vie de l'outil, la rugosité superficielle et les forces de coupe. Les meilleurs résultats ont été obtenus pour les outils PCD [59].

Olsen et al. ont étudié la microstructure et les propriétés physiques et mécaniques du film épais de diamant produit par déposition chimique en phase vapeur (CVD) en vue de son utilisation comme revêtement des outils de coupe. L'introduction du diamant, électriquement conducteur, dans le recouvrement CVD permettra de résoudre quelques problèmes qui limitent la fabrication. On pourra par conséquent s'attendre à sa plus grande pénétration sur le marché et la possibilité de le substituer au PCD [54].

Turchetta et al. ont étudié le comportement des outils revêtus de diamant par déposition chimique en phase vapeur (CVD), en le comparant avec celui des carbures en tungstène traditionnel. Ils ont conclu que les CVD permettaient de réduire significativement la force de coupe et la valeur de l'énergie mise en jeu, et par conséquent il était possible d'augmenter la vitesse de coupe sans augmentation des contraintes dans l'outil [60].

Arumugam et al. ont conduit une recherche sur le comportement des outils avec recouvrement de diamant produit par déposition chimique en phase vapeur (CVD) en comparant, dans le cas du chariotage sec d'alliages d'aluminium renforcés, les outils polis en carbures revêtus avec diamant CVD avec les outils sans polissage. Les outils CVD polis ont eu un meilleur comportement en termes de réduction de l'usure de l'outil (le polissage améliore ainsi la vie de l'outil et réduit les forces de coupe) et d'amélioration de la finition superficielle de la pièce [61].

Fukui et al. ont développé un autre travail semblable pour analyser le comportement d'outils revêtus au DLC (Diamond-Like Carbon) dans l'usinage sec d'alliages d'aluminium [62].

Cabral et al. ont fait des recherches sur l'aptitude d'usinage des outils revêtus de diamant produit par le procédé TMCVD ( Time-Modulated Vapour Chemical Deposition process) en chariotage du graphite et ont comparé les résultats obtenus avec ceux du diamant polycrystallin commercial (PCD). Ils ont démontré que les outils revêtus de diamant TMCVD ont une plus grande résistance à l'usure que les outils revêtus par des techniques conventionnelles ou les outils commerciaux PCD. La taille fine des particules de diamant engendre une plus petite rugosité superficielle et de plus faibles contraintes résiduelles en accordant un meilleur comportement au TMCVD lorsqu'il est comparé avec le diamant conventionnel et avec le PCD [63].

Köpf et al. ont fait des recherches sur des outils revêtus de diamant pour des applications d'usinage de graphite, de matières plastiques renforcées avec des fibres et des alliages d'aluminium, et ont conclu qu'il est possible d'utiliser le diamant revêtu avec un comportement acceptable et potentiel sur le marché des outils [58].

Les outils revêtus de diamant, par déposition chimique en phase vapeur (CVD), peuvent clairement être une alternative économique aux outils de diamant polycrystallin (PCD) afin d'usiner les matériaux avancés [57]. 


\section{Critères d'usinage}

Différents critères ont été avancés afin d'évaluer l'usinage dans le cas des matériaux composites; cependant, il n'existe aucun indice d'usinage qui soit universel. La rugosité et la force spécifique de coupe sont deux critères importants pour évaluer l'usinage d'un matériau composite [46]. La rugosité est largement utilisée comme indice de qualité du produit et dans beaucoup de cas comme spécification technique au niveau des composants mécaniques. La force spécifique de coupe fournit une indication de l'efficacité du processus. Pour obtenir une faible force spécifique de coupe il est nécessaire d'utiliser des outils de diamant polycrystallin (PCD).

Bien que l'usinabilité doit généralement être associée au matériau à travailler, il est nécessaire de souligner que les paramètres fonctionnels de coupe, les outils et le taux d'opération d'usinage sont aussi des facteurs importants qui affectent le processus d'usinage [16].

\subsection{Force et puissance de coupe}

La valeur relative des forces de coupe a une importance cruciale, puisqu'elle affecte la vie des outils. Les forces de coupe influencent naturellement les propriétés mécaniques du matériau usiné (en particulier sa dureté). Dans le cas des composites, elles dépendent du pourcentage volumique des fibres, de leur type et de leur orientation ainsi que des caractéristiques de l'outil de coupe (matériau, géométrie, état de l'outil, etc.).

Quant à la puissance de coupe, elle fournit une information sur la quantité d'énergie à fournir durant le processus d'usinage pour parvenir à éliminer le matériau en excès et atteindre la finition souhaitée (rugosité, précision dimensionnelle, tolérances). Pour cette raison, il est important de réduire la valeur de la puissance de coupe, ce qui implique, logiquement, de réduire les forces de coupe, et requiert d'intervenir sur les paramètres fonctionnels de coupe et sur l'outil. Une plus petite consommation d'énergie se traduit en effet par un plus petit coût de production.

La force spécifique de coupe est un autre indicateur qui est utilisé. Elle est obtenue à partir des forces de coupe et elle est très utile pour évaluer l'efficacité du processus d'usinage. Sreejith et al. ont effectué une étude sur l'effet de la force spécifique de coupe durant le processus d'usinage des matériaux composites à matrice polymère en utilisant des outils de carbures de tungstène. En analysant les signaux d'émission acoustique produits pendant l'usinage, ils ont identifié l'existence d'une vitesse critique d'usinage. L'étude a permis de déterminer aussi la gamme de températures pour lesquelles la pression spécifique de coupe restait stable [64].

\subsection{Usure de l'outil}

Il existe beaucoup de différences concernant les durées de vie des outils de coupe lors de l'usinage des matériaux thermoplastiques. La durée de vie est essentiellement fonction de la nature de l'outil de coupe utilisé : carbures de tungstène ou diamants polycrystallins. Tout le monde est d'accord cependant sur le fait que la plus grande durée de vie des outils est atteinte par les PCD [50]. L'usure de l'outil est utilisée comme critère d'usinage dans le cas des métaux, des matériaux composites à matrice métallique et des céramiques [65-68], dans le cas particulier des matériaux composites à matrice polymérique, spécialement pour les outils de diamant polycrystallin. Dans le cas des outils avec revêtement de diamant ou bien des carbures de tungstène, qui sont des matériaux à haute résistance à l'usure, les essais capables de révéler de manière exacte la durée de vie sont difficiles à envisager. L'usure est par contre plus facile à mettre en évidence quand on utilisera les autres types d'outils conventionnels.

L'usure caractéristique des outils qui est produite par l'usinage des matériaux composites à matrice polymère peut être décrite comme étant essentiellement la superposition de deux mécanismes. Les fibres, avec leur dureté relative importante, sont à l'origine de l'usure abrasive, par contre la matrice plastique présente des propriétés d'adhésion extraordinaires qui peuvent être à l'origine du phénomène de délaminage et la réduction des bords coupants de l'outil [58].

\subsection{Finition superficielle}

Actuellement, on constate une croissance au niveau de la demande sur les composants fabriqués avec des matières plastiques renforcées avec des fibres, des exigences accrues sont aussi formulées au niveau de la précision dimensionnelle et de la finition superficielle souhaitées [69-71]. L'état de surface constitue un paramètre très utile dans la pratique en vue de caractériser les matériaux usinés. La rugosité superficielle est un paramètre qui a une grande influence sur le comportement et le fonctionnement en service des composants mécaniques, et sur le coût de production [72,73]. Elle constitue ainsi un paramètre fondamental dans le contrôle de la qualité. La rugosité est critique dans les contacts mécaniques, la circulation des fluides et des applications semi-conductrices.

Dans la majorité des applications on demande aujourd'hui une grande qualité des surfaces usinées ainsi qu'une exactitude dimensionnelle irréprochable et une intégrité superficielle optimale. Pour cette raison, diverses recherches ont été effectuées dans le but d'optimiser les paramètres de coupe, afin d'obtenir une rugosité spécifiée [41,72]. Ces études ont démontré que la rugosité et le profil des surfaces usinées dépendent dans une grande mesure de l'orientation des fibres, de leur type et de la direction de mesure adoptée pour la rugosité [50,74,75]. La rugosité des surfaces usinées est plus sensible aux variations de l'angle d'enroulement des fibres qu'aux variations de la vitesse d'avance de l'outil [69].

Pour obtenir la rugosité souhaitée il est nécessaire de connaître les mécanismes de coupe et de décollement du matériau et la cinétique du processus d'usinage qui affectent le comportement des outils de coupe [43]. En 
général, la perte des outils de coupe se produit par l'usure progressive ou par rupture. Le degré d'usure de l'outil affecte la qualité des surfaces usinées. Jahanmir et al. [50], Spur et Wunsch [69], ont étudié le processus de chariotage des matières plastiques renforcées avec des fibres de verre (GFRP avec matrice polyester et résine époxy). Ils ont trouvé que la rugosité augmente au fur et à mesure de l'augmentation de la vitesse d'avance et qu'elle ne dépend pas de la vitesse de coupe. Au contraire, Santhanakrishman et al. [76], Ramulu et al. [77], ont obtenu une plus grande qualité superficielle en augmentant la vitesse de coupe. Dans tous les cas, il s'agit là d'un secteur avec de nombreuses questions auxquelles il faudra apporter des réponses.

Kopac et Bahor ont mené une recherche où ils ont analysé l'influence des paramètres d'usinage sur la rugosité. Ils ont utilisé un plan d'expérience et l'analyse de variance pour évaluer cette interaction dans le cas du chariotage des métaux. Cette méthodologie expérimentale a été aussi appliquée par d'autres chercheurs dans l'usinage des matières plastiques renforcées [78].

Bernardos et Vosniakos ont présenté différentes méthodologies pratiques qu'ils ont employées pour prédire la rugosité superficielle (plan d'expériences, intelligence artificielle, etc.). Ils ont considéré l'influence sur la rugosité des paramètres d'usinage, des propriétés des outils de coupe, des propriétés de la pièce [46]. Différents travaux ont été développés dans le domaine du chariotage des matières plastiques renforcées avec des fibres (FRP's) en utilisant des outils de diamant polycrystallin. Ces travaux aboutissent à la conclusion que la rugosité superficielle augmente avec la vitesse d'avance et qu'elle diminue avec la vitesse de coupe. Une des propriétés particulières des FRP's est leur anisotropie. Différents auteurs ont étudié comment l'orientation de la fibre peut influencer la qualité des surfaces usinées, comment influence-telle l'usure de l'outil. De manière générale, quand les fibres se trouvent coupées par traction, on obtient une rugosité et des forces de coupe plus faibles bien que le rapport d'usure de l'outil augmente. Au contraire, la rugosité et forces de coupe augmentent si les fibres sont coupées par cisaillement $[2,26]$.

Eriksen a développé une étude sur l'influence des paramètres de production sur la rugosité superficielle dans l'usinage des thermoplastiques renforcés avec des fibres courtes et il a trouvé que l'effet de l'orientation des fibres est pratiquement insignifiant sur la rugosité [41]. Toutefois, Wang et Zhang ont développé une recherche expérimentale sur la coupe orthogonale des matières plastiques renforcées avec des fibres unidirectionnelles et ont conclu que la rugosité superficielle, la couche endommagée sous la surface et les forces de coupe changent radicalement avec l'orientation de la fibre. L'angle d'orientation de la fibre $\theta$, constitue le facteur clé qui détermine l'intégrité superficielle des composants usinés. $\theta=90^{\circ}$ est l'angle critique, au-delà duquel un endommagement très grave du produit a lieu sous la surface usinée [79]. Chambers et Bishop ont effectué des recherches sur l'usinage des matières plastiques renforcées avec des fibres
(FRP's) et sur le composite carbone/PEEK lorsque différents outils de coupe sont utilisés, comme les carbures de tungstène ou le diamant polycrystallin (PCD). Ils ont conclu que l'outil de coupe PCD présente le meilleur comportement global [49].

Davim et Reis ont présenté une étude sur l'influence des paramètres de coupe (vitesses de coupe et avance) sur la rugosité et sur la précision dimensionnelle en chariotage des tubes de matières plastiques renforcées avec des fibres (FRP's). Ils ont utilisé des matrices orthogonales et l'analyse de variance (ANOVA) pour faire des recherches sur les caractéristiques de coupe des FRP's en utilisant des outils de carbures de tungstène (K15). L'objectif était d'établir des corrélations entre d'une part les paramètres de coupe et d'autre part la rugosité et la précision dimensionnelle pour les pièces de FRP's. En fonction des paramètres de coupe utilisés, il a été possible d'obtenir des surfaces avec une précision dimensionnelle adéquate pour la construction mécanique, IT 9 et 10 [81].

Sreejith et al. ont évalué le comportement des outils de diamant polycrystallin (PCD) pendant le processus d'usinage du composite ablatif constitué de renforts réfractaires tel que le carbone et d'une matrice ablative à base de résine phénolique et ont analysé l'influence du matériau sur l'état final des surfaces usinées. Ils ont observé une vitesse critique de $300 \mathrm{~m} \cdot \mathrm{min}^{-1}$ et un intervalle critique des températures allant de $300{ }^{\circ} \mathrm{C}$ à $350{ }^{\circ} \mathrm{C}$. En se basant sur des essais d'usure de l'outil, ils ont établi que l'outil PCD peut être utilisé de manière économique dans le chariotage des matières plastiques renforcées avec des fibres, puisqu'il a une vie plus grande et fournit une meilleure qualité superficielle sur les pièces de travail [43].

L'analyse expérimentale multi-facteurs a permis de développer des cartes pour le contrôle des surfaces chariotées dans le cas des métaux [81]. Dans d'autres travaux différentes autres techniques sont appliquées pour prédire la rugosité à partir des forces de coupe, en recourant à des algorithmes génétiques ou à des réseaux neuronaux [82-86]. Ces techniques peuvent aussi être appliquées aux matières plastiques renforcées afin d'étudier l'influence des conditions de coupe sur la valeur de la rugosité et de prédire les valeurs à spécifier face à des conditions de travail préétablies.

\section{Conclusion}

Les matériaux composites à base de matrice polymère sont des matériaux de hautes performances. Grâce à leurs excellentes propriétés et leur faible poids, qui est très avantageux par rapport aux matériaux conventionnels et d'autres matériaux composites, ils sont utilisés dans plusieurs applications et dans différents secteurs de la production industrielle. Ceci explique la recherche très active dans ce domaine, et particulièrement celle qui se consacre à l'étude de l'usinabilité de ces matériaux composites.

Puisque l'objectif de la recherche se concentre actuellement sur la définition des modèles physiques et statistiques adéquats permettant de procéder à l'usinage de 
ce type de matériaux dans les meilleures conditions possibles, une vaste revue bibliographique a été effectuée dans ce travail afin de faire le point sur les modèles de coupe qui ont été développés et sur les différents aspects techniques liés à l'usinage des composites à base de matrice polymérique. Dans ce contexte, une analyse des facteurs qui influencent l'usinage et des critères d'usinabilité utilisés pour ces matériaux a été présentée, en insistant en particulier sur les forces de coupe et sur l'état de finition superficielle.

En ce qui concerne les outils de coupe, les expériences montrent en général que les outils de diamant polycrystallin (PCD) permettent d'obtenir les meilleurs résultats possibles (faible consommation d'énergie et finition superficielle optimale). Par ailleurs, il a été souligné que l'usinage de ces matériaux doit être effectué sans refroidissement, ce qui restreint a priori le choix des paramètres fonctionnels de ce processus de fabrication.

On a mis en évidence d'autre part que, pour satisfaire les exigences des diverses applications en matière d'usinage des composites à base de matrice polymérique, il est recommandé de travailler avec des faibles vitesses d'avance et une vitesse de coupe moyenne.

Les premières investigations dans le domaine de l'usinabilité des composites à matrice polymère ont été consacrées principalement à l'étude de l'usinage des matériaux à matrice thermodurcissable renforcée avec des fibres longues, et non pas avec des fibres courtes. L'objectif de la recherche menée dans ce travail était de fournir des résultats et des conclusions sur l'usinage des matériaux composites à matrice thermoplastique renforcée avec des fibres courtes. Des modèles de coupe ont été établis en appliquant des critères classiques comme celui de Merchant. Ce critère s'adapte relativement bien au cas considéré ici à cause de la petite taille des fibres de renforcement et de l'apparition des déformations plastiques du matériau au cours du processus de chariotage qui permet la formation continue de copeau. Ceci permettra dans l'avenir de projeter d'autres travaux expérimentaux afin d'affiner les modèles de coupe, en prenant en considération toutes les connaissances acquises et tous les résultats expérimentaux significatifs dans ce domaine.

La revue bibliographique a été nourrie d'articles scientifiques connus à l'échelle internationale qui relatent l'état de l'art et des connaissances techniques spécifiques au chariotage des matières plastiques renforcées par des fibres.

\section{Références}

[1] G. Petropoulos, C. Pandazaras, Evaluating the real profile length in turning of carbon steels, Ind. Lubr. Tribol. 55 (2003) 128-136

[2] N. Bhatnagar, N. Ramakrishnan, N.K. Naik, On the machining of fiber reinforced plastics (FRP) composite laminates, Int. J. Mach. Tool. Manuf. 35 (1995) 701-716

[3] H. Voss, K. Friedrich, On the wear behaviour of shortfibre-reinforced PEEK composites, Wear 116 (1987) 1-18
[4] R. Krishnamurthy, G. Santhanakrishnan, S.K. Malhotra, Machining of Polymeric composites, Proceedings of the Machining of Composite Materials Symposium, ASM Materials Week (1992) 139-148

[5] M. Rahman, S. Ramakrishna, J.R.S. Prakash, D.C.G. Tan, Machinability study of carbon fiber reinforced composite, J. Mat. Proc. Technol. 89-99 (1999-a) 292-297

[6] M. Rahman, S. Ramakrisna, H.C. Thoo, Machinability study of carbon/PEEK composites, Machin. Science Technology 3 (1999-b) 49-59

[7] R. Komanduri, Machining fiber-reinforced composites, Mech. Eng. 115 (1993) 58-64

[8] Z. Lu, T. Yoneyama, Micro cutting in the micro lathe turning system, Int. J. Mach. Tool. Manuf. 339 (1999) 1171-1183

[9] Y.N. Picard, D.P. Adams, M.J. Vasile, M.B. Ritchey, Focused ion beam-shaped microtools for ultra-precision machining of cylindrical components, Precision Engineering 27 (2003) 59-69

[10] A. Spencer, Deformations of fiber-reinforced materials, Clarendon Press, Oxford, 1972

[11] D. Arola, M. Ramulu, Orthogonal cutting of fiberreinforced composites: A finite element analysis, Int. J. Mech. Sci. 39 (1997) 597-613

[12] G. Caprino, V. Tagliaferri, Damage development in drilling glass-fiber-reinforced plastics, Int. J. Mach. Tool. Manuf. 35 (1995) 817-829

[13] G. Caprino, L. Santo, L. Nele, Interpretation of size effect in orthogonal machining of composite materials. Part I: Unidirectional glass-fiber-reinforced plastics, Composites part A - Applied Science and Manufacturing 29 (1998) 887-892

[14] J. Mathew, N. Ramakrishnan, N.K. Naik, Investigations into the effect of geometry of a trepanning tool on thrust and torque during drilling of GFRP composites, J. Mat. Proc. Tech. 91 (1999-a) 1-11

[15] J. Mathew, N. Ramakrishnan, N.K. Naik, Trepanning on unidirectional composites: delamination studies, Composites Part A: Applied Science and Manufacturing (Incorporating Composites and Composites Manufacturing) 30 (1999-b) 951-959

[16] M.P. Groover, Fundamentals of Modern Manufacturing Materials, Process and Systems, Prentice Hall International Editions, 1996

[17] S.O. An, E.S. Lee, S.L. Noh, A study on the cutting characteristics of glass fiber reinforced plastics with respect to tool materials and geometries, J. Mat. Proc. Technol. 68 (1997) 60-67

[18] J.R. Ferreira, N.L. Coppini, F. Levy Neto, Characteristics of carbon-carbon composite turning, J. Mat. Proc. Technol. 109 (2001) 65-71

[19] H. Hocheng, C.C. Tsao, The path towards delaminationfree drilling of composite materials, J. Mat. Proc. Technol. 167 (2005) 251-264

[20] H. Hocheng, C.C. Tsao, Effects of special drill bits on drilling-induced delamination of composite materials, Int. J. Mach. Tool. Manuf. 46 (2006) 1403-1416

[21] A. Koplev, A. Lystrup, T. Vorm, The cutting process, chips and cutting forces in machining CFRP, Composites 14 (1983) 371-376

[22] C.S. Chang, Turning of glass-fiber reinforced plastics materials with chamfered main cutting edge carbide tools, J. Mat. Proc. Technol. 180 (2006) 117-129 
[23] G.C. Everstine, T.G. Rogers, A Theory of Machining of Reinforced Materials, J. Composi. Mater. 5 (1971) 94-106

[24] A. Koplev, Cutting of CFRP with single edge tools, 3rd International Conference on composite Materials, Paris, 1980

[25] K. Sakuma, M. Seto, Tool wear in cutting glass-fiberreinforced plastics. The relation between fiber orientation and tool wear, Bull. JSME 26 (1983) 1420-1427

[26] H. Takeyama, N. Iijima, Machinability of glass fiber reinforced plastics and applications of ultrasonic machining, Annals CIRP 37 (1988) 93-96

[27] M. Ramesh, K. Seetharamu, N. Ganesan, M. Sivakumar, Analysis of machining of FRPs using FEM, Int. J. Mach. Tool. Manuf. 38 (1998) 1531-1549

[28] M.V. Ribeiro, N.L. Coppini, An applied database system for the optimisation of cutting conditions and tool selection, J. Mat. Proc. Technol. 92-93 (1999) 371-374

[29] M. Mahdi, L. Zhang, An adaptive three-dimensional finite element algorithm for the orthogonal cutting of composite materials, J. Mat. Proc. Technol. 113 (2001-a) 368-372

[30] M. Mahdi, L. Zhang, A finite element model for the orthogonal cutting of fiber-reinforced composite materials, J. Mat. Proc. Technol. 113 (2001-b) 373-377

[31] X.M. Wang, L.C. Zhang, Orthogonal cutting mechanisms of graphite/epoxy composite. Part I: unidirectional laminate, Int. J. Mach. Tool. Manuf. 35 (1995-a) 1623-1638

[32] X.M. Wang, L.C. Zhang, Orthogonal cutting mechanisms of graphite/epoxy composite. Part II: multi-directional laminate, Int. J. Mach. Tool. Manuf. 35 (1995-b) 16391648

[33] T. Kaneeda, CFRP cutting mechanism, Trans. Amer. Manufacturing Res. Inst. SME 19 (1991) 216-221

[34] G. Byrne, U.E. Wunsch, Composite materials in manufacturing engineering, Technical development and applications, NBST (1986)

[35] M. Ramulu, D. Kim, G. Choi, Frequency analysis and characterization in orthogonal cutting of glass fiber reinforced composites, Composites Part A: Applied Science and Manufacturing (Incorporating Composites and Composites Manufacturing) 183 (2003) 949-962

[36] M. Ramulu, S.-Y. Kuo, Y.-M. Chen, D. Kim, R. Spitsen, Cutting characteristics of preform and SMC composites, Transactions of the North American Manufacturing Research Institute of SME 32 (2004) 239-246

[37] U.A. El-Sonbaty, T. Khashaba, T. Machaly, Factors affecting the machinability of GFR/epoxy composites, Compos. Struct. 63 (2004) 329-338

[38] J.R. Ferreira, N.L. Coppini, G.W.A. Miranda, Machining optimisation in carbon fiber reinforced composite materials, J. Mat. Proc. Technol. 92 (1999) 135-140

[39] W.H. Yang, Y.S. Tarng, Design optimization of cutting parameters for turning operations based on the Taguchi Method, J. Mat. Proc. Technol. 84 (1998) 122-129

[40] R. Varatharajan, S.K. Malhotra, L. Vijayaraghavan, R. Krishnamurthy, Mechanical and machining characteristics of GF/PP and GF/Polyester composites, Materials Science and Engineering B 132 (2006) 134-137

[41] E. Eriksen, Influence from production parameters on the surface roughness of a machined short fibre reinforced thermoplastic, Int. J. Mach. Tool. Manuf. 39 (1999) 16611618

[42] B.Y. Lee, Y.S. Tarng, H.R. Lii, An Investigation of Modelling of the Machining Database in Turning Operations, J. Mat. Proc. Technol. 105 (2000) 1-6
[43] P.S. Sreejith, R. Krishnamurthy, S.K. Malhota, K. Narayanasamy, Evaluation of PCD tool performance during machining of carbon/phenolic ablative composites, J. Mat. Proc. Technol. 104 (2000) 53-58

[44] R.Q. Sardinas, P. Reis, J.P. Davim, Multi-objective optimization of cutting parameters for drilling laminate composite materials by using genetic algorithms, Composites Science and Technology 66 (2006) 3083-3088

[45] A. Sang-Olk, L. Eun-Sang, N. Sang-Lay, A study on the cutting characteristics of glass fiber reinforced plastics with respect to tool materials and geometries, J. Mat. Proc. Technol. 68 (1997) 60-67

[46] P.G. Bernardos, C.G. Vosniakos, Predicting surface roughness in machining: a review, Int. J. Mach. Tool. Manuf. 43 (2003) 833-844

[47] I. Reineck, M.E. Sjöstrand, J. Karner, M. Pedrazzini, Diamond coated cutting tools, Int. J. Refractory Metals and Hard Materials (1996) 187-193

[48] Q.S. Bai, Y.X. Tao, P. Bex, G. Zhang, Study on wear mechanisms and grain effects of PCD tool in machining laminated flooring, Int. J. Refractory Metals and Hard Materials 22 (2004) 111-115

[49] A. Chambers, G. Bishop, The drilling of carbon fibre polymer matrix composites, Processing and Manufacturing 3 (1995) 565-572

[50] S. Jahanmir, M. Ramulu, P. Koshy, Machining of ceramics and composites, Marcel Dekker Inc., 1998, pp. 238243

[51] C. Faure, W. Hänni, C.J. Schmutz, M. Gervanoni, Diamond-coated tools, Diam. Rel. Mat. 8 (1999) 830-833

[52] J.Y. Sheikh-Ahmad, J.S. Stewart, H. Feld, Failure characteristics of diamond-coated carbides in machining woodbased composites, Wear 255 (2003) 1433-1437

[53] M. Belmonte, F.J. Oliveira, M.A. Lanna, C.M. Silva, E.J. Corat, R.F. Silva, Turning of CFRC composites using Si3N4 and thin CVD diamond coated Si3N4 tools, Materials Science Forum 455-456 (2004) 609-613

[54] R.H. Olsen, R.C. Dewes, D.K. Aspinwall, Machining of electrically conductive CVD diamond tool blanks using EDM, J. Mat. Proc. Technol. 149 (2004) 627-632

[55] R.S. Sussmann, J.R. Brandon, S.E. Coe, C.S.J. Pickles, C.G. Sweeney, A. Wasenczuk, C.J.H. Wort, C.N. Dodge, CVD diamond: new engineering material for thermal, dielectric and optical applications, Ind. Diamond Rev. 3 (1998) 69-77

[56] S. Kalpakjian, S.R. Schmid, Manufactura, ingeniería y tecnología, Pearson Educación, México, 2002

[57] Y. Kevin Chou, J. Lui, CVD diamond tool performance in metal matrix composite machining, Surf. Coat. Technol. 200 (2005) 1872-1878

[58] A. Köpf, S. Feistritzer, K. Udier, Diamond coated cutting tools for machining of non-ferrous metals and fibre reinforced polymers, Int. J. Ref. Met. Hard Mater. 24 (2006) 354-359

[59] J.P. Davim, Diamond tool performance in machining metal-matrix composites, J. Mat. Proc. Technol. 128 (2002) 100-105

[60] S. Turchetta, L. Carrino, W. Polini, CVD diamond insert in stone cutting, Diam. Rel. Mat. 14 (2005) 641-645

[61] P.U. Arumugam, A.P. Malshe, S.A. Batzer, Dry machining of aluminium silicon alloy using polished CVD diamond-coated cutting tools inserts, Surf. Coat. Technol. 200 (2006) 3399-3403 
[62] H. Fukui, J. Okida, N. Omori, H. Moriguchi, K. Tsuda, Cutting performance of DLC coated tools in dry machining aluminium alloys, Surf. Coat. Technol. 187 (2004) $70-76$

[63] G. Cabral, P. Reis, R. Polini, E. Titus, N. Ali, J.P. Davim, J. Grácio, Cutting performance of time-modulated chemical vapour deposited diamond coated tool inserts during machining graphite, Diam. Rel. Mat. (2006) 1753-1758

[64] P.S. Sreejith, R. Krishnamurthy, S.K. Malhotra, Effect of specific cutting pressure and temperature during machining of carbon/phenolic ablative composite using PCBN, J. Mat. Proc. Technol. 183 (2007) 88-95

[65] H.V. Ravindra, Y.G. Srinivasa, R. Krishnamurthy, Tool wear monitoring in turning using a pattern-recognition technique, J. Mat. Proc. Technol. 37 (1993-a) 731-740

[66] H.V. Ravindra, Y.G. Srinivasa, R. Krishnamurthy, Modeling of tool wear based on cutting forces in turning, Wear 169 (1993-b) 25-32

[67] M.E.R. Bonifacio, A.E. Diniz, Correlating tool wear, tool life, surface roughness and tool vibration in finish turning with coated carbide tools, Wear 173 (1994) 137-144

[68] S. Dolinsek, J. Kopac, Acoustic emission signals for tool wear identification, Wear 225 (1999) 295-303

[69] G. Spur, U.E. Wunsch, Turning of Fiber-Reinforced Plastics, Manuf. Rev. 1 (1988) 124-129

[70] K. Palanikumar, L. Karunamoorthy, R. Karthikeyan, Optimizing the machining parameters for minimum surface roughness in turning of GFRP composites using design of experiments, J. Mat. Sci. Technol. 20 (2004) 373378

[71] K. Palanikumar, Cutting parameters optimization for surface roughness in machining of GFRP composites using Taguchi's method, J. Reinf. Plast. Compos. 25 (2006) 1739-1751

[72] O.B. Abouelatta, J. Mádl, Surface roughness prediction based on cutting parameters and tool vibrations in turning operations, J. Mat. Proc. Technol. 118 (2001) 269277

[73] G. Petropoulos, J.P. Davim, F. Mata, C. Pandazaras, New considerations of evaluating the anisotropy of machined surfaces, J. Balkan Tribological Association 12 (2006a) $1-6$

[74] M. Ramulu, C.W. Wern, J.L. Garbini, Effect of the direction on surface roughness measurements of machined graphite/epoxy composite, Compos. Manuf. 4 (1993) 39-51

[75] A.A. Cenna, P. Mathew, Evaluation of cut quality of fibre-reinforced plastics, Int. J. Mach. Tool. Manuf. (1997) $723-736$
[76] G. Santhanakrishman, R. Krishnamurthy, S.K. Malhota, Machinability Characteristics of Fiber Reinforced Plastics Composites, J. Mech. Work. Technol. (1988) 195-204

[77] M. Ramulu, D. Arola, K. Colligan, Preliminary Investigation of Effects on the Surface Integrity of Fiber Reinforced Plastics, PD-VOL-64-2, Engineering Systems Design and Analysis 2 ASME (1994) 93-101

[78] J. Kopac, M. Bahor, Interaction of the technological history of a workpiece material and the machining parameters on the desired quality of the surface roughness of a product, J. Mat. Proc. Technol. 92-93 (1999) 381-387

[79] X.M. Wang, L.C. Zhang, An experimental investigation into the orthogonal cutting of unidirectional fiber reinforced plastics, Int. J. Mach. Tool. Manuf. 43 (2003) 10151022

[80] J.P. Davim, P. Reis, Dimensional precision and surface roughness on turning tubes in fibre reinforced plastics based on the design experiments, Int. J. Mat. Prod. Technol. 20 (2004-a) 268-279

[81] G.P. Petropoulos, C.N. Pandazaras, N.M. Vaxevanidis, A. Antoniadis, Multi-parameter identification and control of turned surface textures, Int. J. Adv. Manuf. Technol. 29 (2006-b) 118-128

[82] K.A. Risbood, U.S. Dixit, A.D. Sahasrabudhe, Prediction of surface roughness and dimensional deviation by measuring cutting forces and vibrations in turning process, J. Mat. Proc. Technol. 132 (2003) 203-214

[83] M. Brezocnik, M. Kovacic, M. Ficko, Prediction of surface roughness with genetic programming, J. Mat. Proc. Technol. 157 (2004) 28-34

[84] P.J. Núñez, J. Simao, J.M. Arenas, C. De la Cruz, Surface roughness characterization using cutting force analysis, regression and neural network prediction models, Mat. Sci. Forum 526 (2006) 211-216

[85] K. Palanikumar, L. Karunamoorthy, R. Karthikeyan, Parametric optimization to minimise the surface roughness on the machining of GFRP composites, J. Mat. Sci. Techn. 22 (2006-a) 66-72

[86] K. Palanikumar, L. Karunamoorthy, R. Karthikeyan, Optimization of machining parameters in turning GFRP composites using a carbide (K10) tool based on the Taguchi method with fuzzy logics, Met. Mater. Int. 12 (2006-d) 483-491 